\title{
FUN3D Grid Refinement and Adaptation Studies for the Ares Launch Vehicle
}

\author{
Robert E. Bartels* \\ Veer Vatsa ${ }^{\dagger}$ \\ Jan-Renee Carlson ${ }^{\ddagger}$ \\ Mike Park ${ }^{\S}$ \\ NASA Langley Research Center, Hampton, VA, 23681, USA \\ Raymond E. Mineck ${ }^{\text {II }}$ \\ ViGYAN Inc., Hampton, VA, 23681, USA
}

\begin{abstract}
This paper presents grid refinement and adaptation studies performed in conjunction with computational aeroelastic analyses of the Ares crew launch vehicle (CLV). The unstructured grids used in this analysis were created with GridTool and VGRID while the adaptation was performed using the Computational Fluid Dynamic (CFD) code FUN3D with a feature based adaptation software tool. GridTool was developed by ViGYAN, Inc. while the last three software suites were developed by NASA Langley Research Center. The feature based adaptation software used here operates by aligning control volumes with shock and Mach line structures and by refining/de-refining where necessary. It does not redistribute node points on the surface. This paper assesses the sensitivity of the complex flow field about a launch vehicle to grid refinement. It also assesses the potential of feature based grid adaptation to improve the accuracy of CFD analysis for a complex launch vehicle configuration. The feature based adaptation shows the potential to improve the resolution of shocks and shear layers. Further development of the capability to adapt the boundary layer and surface grids of a tetrahedral grid is required for significant improvements in modeling the flow field.
\end{abstract}

\section{Nomenclature}

$\begin{array}{ll}C_{p} & \text { surface pressure coefficient } \\ M & \text { Mach number } \\ \text { Nnodes } & \text { number of nodes in volume grid } \\ x, y, z & \text { location of point in cartesian coordinates } \\ y^{+} & \text {non-dimensional distance normal to the surface } \\ \alpha & \text { angle of attack, degrees } \\ \phi & \text { clocking angle }\end{array}$

Note to Readers: The predicted performance and certain other features and characteristics of the Ares I and Ares I-X launch vehicles are defined by the U.S. Government to be Sensitive but Unclassfied (SBU). Therefore, details have been removed from all plots and figures and tabulated data may have been rescaled.

\section{Introduction}

The Constellation program along with the Ares and the Orion projects are tasked with developing the crew launch vehicle (CLV) and crew exploration vehicle (CEV) technology necessary to take humans beyond low-earth orbit. The engineering of the Ares CLV is a departure from the past development of launch vehicles. This vehicle will be designed with the smallest ever proportion of aerodynamic data derived from wind tunnel testing and the largest ever component due to computational fluid dynamics. This approach poses challenges in validating methodologies for the

\footnotetext{
*Aerospace Engineer, Aeroelasticity Branch, Senior Member

${ }^{\dagger}$ Aerospace Engineer, Computational Aerosciences Branch, Associate Fellow

*Aerospace Engineer, Computational Aerosciences Branch, Senior Member

${ }^{\S}$ Aerospace Engineer, Computational Aerosciences Branch, Senior Member

ISenior Member
} 
highly complex flow field about a launch vehicle. One of the notable features of the Ares CLV is the use of a five segment Solid Rocket Booster (SRB) as a first stage with a larger diameter upper stage. The two stages are connected by an aft facing frustum. This configuration has the potential of producing flow field separation from the frustum which can significantly influence overall vehicle aerodynamics. Along with the usual geometric complexity of protuberances over a major launch vehicle, this hammer-head configuration poses a challenge to CFD analysis. The response to this challenge has been a wide spread use of high fidelity Navier-Stokes analyses in the Ares project. Furthermore, analyses are being conducted that have never before been attempted at the preliminary and critical design stages of a launch vehicle. Computational aeroelastic analysis using the high fidelity Navier-Stokes analysis tool FUN3D is performed at the preliminary design stage of the Ares I launch vehicle.

The structural integrity of a launch vehicle can be decided by the accuracy of the aerodynamic measurements or modeling. Inaccurate measurement of the buffet levels caused by shock separation or vortex shedding can potentially lead to catastrophic consequences. ${ }^{1,2}$ Data from one of the Titan IVB flights showed larger than expected structural response to aerodynamic buffet. An after the fact CFD analysis of the heavy lift Titan IVB at the specific condition in question, using a 4 million node structured grid, showed that alternate pair shed vortices originating between the frustum and the conical noses of the external boosters were responsible for a higher amplitude buffet environment than was anticipated. ${ }^{2}$

The quality of the aerodynamic simulation is dependent on many factors such as the size and clustering of the computational grid. For good quality solutions it is necessary to have grid points concentrated in regions of large flow field gradients, such as at surface corners or edges or at transonic and supersonic shocks and shock separations. Complex surface features and protuberances may contribute to the difficulty of modeling the flow field. The flow structures resulting from sharply defined surface features will interact with the naturally occurring three dimensional cylinder/body separation and shocks. On the other hand, protuberances and rings also tend to fix separations at sharp corners and edges, and a fortunate consequence of many unstructured grid generation software packages is that even triangular surface edges tend to be aligned with sharp edges and corners.

The Ares I aeroelastic analysis is intended to provide guidance regarding vehicle buffet and flutter stability over the vehicle ascent trajectory. The ascent of a launch vehicle presents it with a rather large range of flight conditions. The transonic Mach number range is of most interest, but even in this limited range, as the Mach number increases, the shock and shock separation will traverse a large extent of the vehicle length. The large extent over which the shock traverses would seem to require multiple grids at different portions of the ascent. An alternative to generating different grids is to use grid adaptation.

Several types of grid adaptation are in use, based on flow field gradient or on an output functional. The output error functional utilizes the dual or adjoint to account for the influence that solution error has on the output of interest. The 2D adjoint and output error methods of Venditti and Darmofal ${ }^{3,4}$ have been extended to the 3D unstructured NavierStokes code FUN3D ${ }^{5}$ This method shows promise in application to inviscid, laminar and turbulent flow problems and has been shown to give excellent results while reducing unnecessary grid count. ${ }^{5-8}$ The adjoint based method has a solid mathematical foundation while the method based on flow gradients does not. The gradient based method has been shown to fail on problems for which the output based method succeeds. Never-the-less one difficulty with the output based method as it is currently typically formulated is that it is memory intensive. This has proven problematic in the present Ares grid adaptation studies because of the size of the grids being used. This limitation of the current implementation of the output based adaptation has led to the exploration of the use of gradient based adaptation for the Ares launch vehicle.

Recent studies have been performed for an atmospheric re-entry vehicle using the FUN3D unstructured NavierStokes solver and several types of gradient based tetrahedral grid refinement. In one scheme, grid movement using the spring analogy based on temperature gradient is used, and a second method using the first derivative of density with anisotropic orientation and scaling determined by the Mach number Hessian. ${ }^{9}$ In that study the flow feature resolved was a single hypersonic bow shock. Either gradient based adaptation technique proved adequate for the relatively modest shock structure. However, a launch vehicle during ascent represents a much more challenging case.

Grid refinement and adaptation studies have been performed for a variety of launch vehicles during ascent. The focus generally has been on a few conditions in the transonic range. ${ }^{10-12}$ The influence of grid refinement with an unstructured Euler code on transonic flow about a multi-stage satellite launch vehicle with multiple boosters was studied. ${ }^{11}$ A comparison with wind tunnel data for multiple transonic conditions showed that one of those conditions $\left(M_{\infty}=0.90\right)$ posed the most difficulty in accurately capturing the correct shock location. This may have been due to the coarseness of the grids (the finest had 200,000 nodes) and inadequate clustering of nodes. A more recent study has been conducted with an unstructured inviscid solver using h-refinement scheme that is sensitive to flow gradients. In this study node adaptation was performed in both the flow field and the launch vehicle body. ${ }^{13} \mathrm{~A}$ hexahedral grid combined with an agglomeration multi-grid has been used by Scalabrin and Azevelo. In that instance, surface and flow field adaptation was for an inviscid calculation only. ${ }^{12}$ 
One of the challenges in adaptation of both off body and surface grids is the adaptation of the high Reynolds number viscous boundary layer. Much improved unstructured high Reynolds number viscous results computed on a hexahedral grid with regular multi-grid refinement have been recently shown for a launch vehicle. ${ }^{14}$ Although launch vehicles with external boosters were modeled in that study, all surfaces were clean and grid clustering at cylinder apex and junctions should have greatly aided capture of flow features.

Flow gradients at the surface that cut across an irregular surface triangulation complicate the adaptation of the surface grid, but in theory adaptive alignment of surface grid edges with flow features can be done much as it is away from surfaces. This capability of triangular element surface adaptation needs additional development. To bypass this issue recent studies have adapted a mid-field sonic boom of a sharp nosed cylindrical body in which the surface topology requires little or no refinement. ${ }^{15}$ A tetrahedral cut cell approach was implemented to permit the adaptation of high-aspect ratio tetrahedral control volumes without the constraint of producing a shock boundary conforming grid.

This paper extends the complexity of the configuration and grid size to which tetrahedral grid adaptation is applied. We explore the effect of refinement and adaptation for a high flight Reynolds number flow about a hammerhead launch vehicle having complex protuberances and rings. The configuration used in the present study is the Ares I a105 outer mold line (OML). A drawing of the exterior surface is presented in Figure 1. This configuration is based on a simplified OML still with over 30 protuberances. Protuberances include launch abort system (LAS) tower nozzles, first and second stage service tunnels. Many of the protuberances are located near or just forward of the frustum. The first stage includes eight aft skirt booster deceleration motor (BDM) housings and four aft skirt tie down flare wedges. Five rings on the upper stage, two rings just aft of the frustum, and SRB stiffener rings near the aft skirt were included. Service panels that protruded less than an inch from the surface were not included.

Sensitivity studies on successively refined grids have been performed to assess the level of grid refinement necessary to capture the salient flow features. One notable result of these refinement studies is the use of grid sizes that have pushed the limit of the available computing and CFD software. In addition, the potential of grid adaptation to improve accuracy and to reduce the computational overhead has been assessed. To accomplish these goals three flight conditions during a nominal ascent trajectory $\left(M_{\infty}=0.50,0.90\right.$ and 1.55$)$ were selected for analysis. These conditions are shown in Table 1. The first condition is expected to be a purely subsonic case. The second of these conditions was chosen as a representative transonic condition because of experience with previous launch vehicles. ${ }^{16}$ Transonic conditions are challenging and interesting from the stand point of buffet. The aeroelastic dynamic response peaks around Mach $1 .{ }^{16}$ It was found, however, that at the third condition (maximum dynamic pressure) there were in fact multiple shocks located in the area of the interstage and frustum while most of the upper stage flow is supersonic. While this localization of the shock suppresses much of the vehicle buffeting response, it is challenging aerodynamically because of the interaction of the shock structures with the protuberances and frustum flow separation. Because of its complexity, the case at maximum dynamic pressure was chosen as the focus of the present paper.

\section{FUN3D Solver and Grid Adaption Software}

The Navier-Stokes code used in this study is the Fully Unstructured Navier-Stokes Three-Dimensional FUN3D code, a finite-volume unstructured CFD code for either compressible or incompressible flows. ${ }^{17,18}$ Flow variables are stored at the vertices of the grid. FUN3D can solve the discrete compressible Euler or Reynolds-averaged NavierStokes (RANS) flow equations either tightly or loosely coupled with a turbulence model on mixed element grids, including tetrahedra, prisms, pyramids and hexahedra. In the present study the RANS solver and the loosely coupled Spalart-Allmaras turbulence model ${ }^{19}$ are used on an all tetrahedron grid. FUN3D employs an implicit upwind algorithm in which the inviscid fluxes are obtained with a flux-splitting scheme. At interfaces delimiting neighboring control volumes, the inviscid fluxes are computed using an approximate Riemann solver based on the values on either side of the interface. Interface values are obtained by a least squares extrapolation using gradients computed at the vertices. Reconstruction requires limiting for flows with strong shocks. A variety of gradient limiters are available. In the present study the low dissipation flux splitting scheme for the inviscid flux construction, and the blended Van Leer flux limiter ${ }^{20}$ were used. For tetrahedral grids the full viscous fluxes are made discrete by using a finite-volume formulation in which the required velocity gradients on the dual faces are computed using the Green-Gauss theorem. ${ }^{17}$ This approach is equivalent to a Galerkin type approximation. Steady state solutions are accelerated to convergence by the use of local time stepping. ${ }^{21}$ Domain decomposition exploits the distributed high-performance computing architectures that are necessary for the grid sizes used in the present study.

Feature based adaptation is performed after obtaining an initial solution. The feature based adaptation request is specified through a grid metric. This 3-by-3 symmetric positive metric is computed at each node of the grid and de- 
couples the request from the grid adaptation mechanics. The options available in FUN3D to formulate the metric are detailed in Bibb et al. ${ }^{9}$ The adaptation mechanics are implemented in a library that is evoked directly from the FUN3D suite of tools. This adaptation library utilizes node insertion, node movement, element collapse, and element connectivity swapping to iteratively drive the grid to a unit grid in the specified grid metric. The adaptation mechanics are executed in the same domain decomposed parallel environment as the flow solver. Details of the metric formulation, adaptation mechanics, and parallel execution scheme are available in Park ${ }^{22}$ and Lee-Rausch et al. ${ }^{5}$

Producing and modifying highly anisotropic grids suitable for simulating high Reynolds number turbulent flows is an extremely challenging task, especially near curved solid wall boundaries. This study avoids the issue of adapting high aspect ratio elements on curved boundaries by utilizing a hybrid approach which freezes the near-wall grid and adapts elements outside this zone. ${ }^{15}$ A hybrid approach simplifies the adaptation mechanics and increases adaptation robustness. This hybrid approach is not applicable to problems with under resolved initial boundary layer grids, because that portion of the grid is held constant during the simulation. However, the hybrid approach is a powerful technique for problems with important off-body anisotropic features, i.e., shocks, expansions, and shear layers.

\section{Grid Refinement Studies}

A study was performed to assess an acceptable level of grid convergence. In the pursuit of this strategy, four grids were developed, designated the extra coarse, coarse, baseline and fine. Table 2 presents details of the four grids. All were generated using the 64-bit version of VGRID 4.00. Nominal circumferential spacing on the baseline grid was $3.1^{\circ}$. Reduced node spacing was used in the vicinity of steps, rings, tunnels, and regions with significant changes in local slope in order to capture the gradients produced by surface curvature. Typical spacing at surface discontinuities, such as protuberance/body junctions, was generally around 0.2 inches on the baseline grid. For a node based unstructured code using a tetrahedral grid this spacing may be considered relatively coarse. However, a sufficient decrease in node spacing at corners would also have driven grid sizes to well over 100 million. Compromising the spacing at geometric corners allowed additional nodes to be placed away from protuberances without significantly increasing the grid count. The intention is to provide better resolution of flow features that are not directly associated with protuberances. Even with the present grids, to resolve the geometric detail of all protuberances required around 28 million nodes out of the 41 million node baseline grid. One of the purposes of the present study is to assess whether this strategy is justified by the flow field complexity.

Figures 2 - 3 show surface details of the four grids. Figure 2 shows the grid over the frustum region. Because of the potential of complex flow, the grid in this area is finer than that over protuberance free surfaces. In Figure 2 is also shown the refinement around the roll control motor and two first stage tumble motors. Most protuberances had both line sources at edges and volume sources for area control. Figure 3 shows aft skirt details and the grid resolution around the SRB stiffener rings, BDMs and flare wedges. The off surface grid in the frustum region is shown in Figure 4. The off surface grid in the aft skirt region is shown in Figure 5. The effect of including both a BDM volume source and line sources around all BDMs can clearly be seen in the finely detailed off surface grid.

For the expected separation and shear layer over the frustum, a conical source was added to the VGRID model extending well into the surrounding flow field. (See Figure 4) The conical source reduced grid spacing in that area significantly. While the conical source does not produce a grid acceptable for resolving a shear layer, it does enrich the off body grid to aid if subsequent grid adaptation is used. Since the region of flow recirculation in front of the aft skirt was not expected to be as large as that over the frustum, the aft skirt does not have a similar conical VGRID source. Comparing Figure 4 with 5 clearly shows the effect of the conical source in the frustum region.

The surface definition for the Ares I configuration had main engine nozzle and all roll and attitude control nozzles closed at the exit. The main engine nozzle surface grid was kept fairly coarse with respect to the rest of the vehicle. Normally the vehicle would have engine exhaust trailing the main nozzle, whereas in this study it does not. Since the wake trailing the vehicle was not the focus of the present study, coarser gridding in that region was used mainly to eliminate the possibility of vortex shedding from the aft end that would destabilize the computation of the rest of the vehicle flow field.

Spacing of the first node from the surface was selected to provide a non-dimensional turbulent wall spacing, $\Delta y^{+}$, of 1.0 or less on most of the vehicle. Growth rate of the node spacing was selected to provide at least 25 nodes in the advancing layers region in an attached boundary layer where viscous effects predominate. For all four grids, the spacing from a surface node to the first node off the surface as well as the growth rate of the node spacing in the advancing layer region were kept constant. This approach was required to limit the size of the finest grid to something less than 100 million nodes.

The refinement of successive grid levels was accomplished by doubling the number of surface nodes, and otherwise 
keeping the boundary layer spacing normal to the vehicle surface unchanged. Regions of grid clustering around all surface features and all volume sources were refined uniformly with the rest of the vehicle. Each of the four grids was partitioned for processing on multiple computer cores. The extra-coarse grid was partitioned for 120 cores, the coarse grid for 240 cores, the baseline grid for 360 cores, and the fine grid for 480 cores.

Grid convergence was assessed at three ascent conditions, Mach 0.5, 0.9 and 1.55. These conditions are shown in Table 1. FUN3D was run at each of these conditions until the solution met the following convergence criteria: the residual in the density equation was reduced by about 3 orders of magnitude and the variation of the longitudinal force and moment coefficients was reduced to less than 1-percent of the average value. Converged FUN3D solutions were obtained for all cases in the grid convergence study except the Mach number 0.90 and 1.55 cases on the fine grid. These cases showed small oscillation in the force and moment coefficients. Inspection of the time history of the surface $C_{p}$ contours on the fine grid revealed that the majority of the oscillation was around the protuberances in the interstage and frustum regions. This factor needs to be kept in mind when observing the difference in $C_{p}$ contours between the fine and coarser grids.

Surface pressure coefficients from the three coarser grids were interpolated onto the fine grid surface and the change in pressure coefficient between that and each of the coarser grids was calculated at each fine grid surface node. A program was used that interpolates the flow variables between FUN3D generated Tecplot ${ }^{23}$ grid surface files consisting of unstructured triangular facets. For every point on the destination grid, the interpolation scheme takes the weighted average of the ten nearest points on the source grid. The weighting function is $1 / r^{3.5}$ where $r$ is the distance between source and destination node point. The 10 nearest points are found using the KDTREE 2 library of Kennel. ${ }^{24}$

Differences in the surface pressure contours for the successively finer grids at the maximum dynamic pressure condition are presented in Figure 6. In areas away from protuberances, and major vehicle diameter changes, all the grids produce nearly the same surface loading. The largest differences, shown in red and blue, are typically near protuberances: near the LAS nozzles, the ogive fairing for the crew exploration vehicle, the lower part of the upper stage, the frustum, and the rings and aft skirt at the base of the first stage. Because the shock has jumped to the frustum region at Mach 1.55, that region presents the greatest sensitivity to grid refinement. In general the difference between the pressure coefficient of the fine and coarser grids diminishes with each successively finer grid, however, it is not clear that convergence has been achieved even with the finest grid used here.

Since the majority of the difference in results is in the frustum and aft skirt areas, the remaining figures will concentrate on these regions. The surface streamlines over the frustum, shown in Figure 7, indicate a complex flow pattern in that region. The deceleration of the flow ahead of the upper stage roll control motor and acceleration of the flow through the gap between it and the first stage tumble motor result in a complex recirculation pattern that changes with each successive grid. The swirling flow around the side of the first stage tumble motor widens and the accelerated flow around the roll control motor changes shape with each successive grid.

Comparison of the surface streamlines on the lower portion of the first stage for the four grids is presented in Figure 8 . The extra-coarse and coarse grids generally fail to capture the complex recirculation between the large rings whereas the baseline and fine grids generally capture these features. The streamlines over the aft skirt also appear to be better defined with the baseline and fine grids.

Figures 9 to 13 focus on the off body flow in the frustum region. The circumferential flow cuts of Figure 9 show contours of negative streamwise velocity. This effectively shows the complex pattern of three dimensional recirculation developing between the upper stage roll control motor and first stage tumble motor. The shape and location of the regions of recirulation change with each grid. The reduction in the size of the recirculation pattern is due to a better refinement of the accelerating flow between protuberances with each successive grid.

Data was extracted from the solution in the area of the frustum at a succession of clocking angles. The orientation of the clocking angles is shown in Figure 10. Figures 11 to 13 present flow field cuts at clocking angles of 30, 90 and 180 degrees. The contours are of velocity in the streamwise direction. The reason for the complexity of the surface streamlines and sensitivity to grid refinement, shown in Figure 7, becomes clear in the present figures. The frustum flow field shows the development of a supersonic acceleration around the interstage to frustum junction terminating in a shock separation.

Depending on the local geometry, the supersonic acceleration and shock either enhances the separation or retards it. The flow patterns at each clocking angle significantly changes with refinement. At some angles the flow separation deepens with refinement of the grid, as seen in Figure 12. At other angles, where supersonic acceleration with a terminating shock occurs, the fine grid produces a reattachment of the boundary layer and a much smaller recirculation zone. This is seen in Figures 11 and 13. This alternating pattern of separation and reattachment due to supersonic acceleration around the interstage/frustum junction is the reason for the complex flow patterns in this region at Mach 1.55. By comparing successively refined solutions it is also clear that additional grid refinement between the interstage/frustum junction and the first stage tumble motors changes the location of the shock separation relative to the stagnation ahead of the protuberance. 
Figure 14 shows flow field contours of negative values of the streamwise component of velocity. By plotting contours of negative streamwise velocity only, regions of recirculating flow are highlighted. The solutions on successively refined grids indicate a significant change in the depth and location of the regions of flow recirculation. The extra coarse grid produces almost no flow recirculation, whereas the baseline and fine grids show a rather deep region of recirculation. The locations of the recirculating flow regions change due to a sensitivity of the alignment of vortical structures with grid spacing.

\section{Feature Based Adaptation}

The FUN3D software includes an option for feature-based grid adaptation ${ }^{9}$. Feature based grid adaptation can potentially add a significant number of node points to resolve flow features. The present grid refinement studies show that the coarse grid was suitable for large regions on the first and second stages where there were few protuberances. Since all the grids receive significant grid refinement in the frustum and aft skirt areas it was postulated that an adapted coarse grid with refined off-body node spacing might resolve flow field features in the frustum and aft skirt regions that were found only on the baseline or fine grids. It is also hoped that the present grid adaptation strategy using adaptation of the coarse grid with primarily grid realignment would offer some improvement without the burden of a significant addition of nodes. This strategy is reflected in the total number of nodes at each of the seven adaptation cycles shown in Table 3.

For this study, cells could be skewed with a maximum 20:1 ratio. Maximum cell size in coarsening was limited to 340 percent of the upper stage diameter. The feature-based adaptation was limited to nodes beyond a 1.3 percent of the upper stage diameter distance above the surface. Limited grid refinement was made in regions where there were gradients in the entropy. Similarly, grid coarsening was implemented in regions with effectively no gradient in entropy. Normally, the grid generation process attempts to fill the region outside of the advancing layers with isotropic tetrahedral elements. For each grid adaptation cycle, four refinement/coarsening iterations were used.

Surface streamlines near the frustum for the original coarse, the adapted coarse and the fine grids are presented in Figure 15. The swirling flow around the side of the first stage tumble motor in the coarse grid solution is significantly different than the solution on the fine grid. The coarse grid also does not predict the accelerated flow between the upper stage roll control motors and first stage tumble motors to the extent that the fine grid does. The location and size of the swirling flow next to the first stage tumble motor as well as the accelerated flow between protuberances are better captured with the adapted grid. However, the surface streamlines of the adapted solution clearly are not fully converged to the fine grid solution.

Figure 16 shows the difference between surface pressure coefficient contours on the adapted coarse and the fine grid, and for comparison, the change in surface pressure coefficient contours between the original coarse and fine grids. The original coarse grid showed large differences between it and the fine grid in the aft skirt and the frustum regions. The aft skirt region shows significant improvement due to grid adaptation while there is only slight to moderate improvement in the frustum and crew module regions.

Off-body flow field slices in the $30^{\circ}$ and $180^{\circ}$ clocking planes of the frustum region are presented in Figures 17 and 18. Figures on the left show the original coarse, adapted and fine grids superimposed on entropy contours. The right side shows Mach contours. The main effect of the grid adaptation is to re-align cell edges with the off surface shocks and shear layers. The shocks are clearly better resolved in the adapted grid. The streamwise extent of the flow recirculation and the location of the shear layer are also better resolved in the adapted grid. Focusing on the fine grid Mach contours, the flow accelerates supersonically around the interstage/frustum junction. The extent of the supersonic region is much larger in the fine grid than the coarse grid solution. This in large part is the cause of the reduced size of the recirculation in the fine grid solution. The adapted coarse grid improves the location of the terminating shock somewhat, but still under predicts the size of the supersonic acceleration. Figure 18 shows the flow field slice at a clocking angle of $180^{\circ}$. The shock structures are again better resolved in the adapted solution. The reattachment of the boundary layer due to supersonic acceleration is again also better modeled with the adapted solution.

Figure 19 shows flow field contours for a cut perpendicular to the axis of the vehicle. Figures on the left show the original coarse, adapted and fine grids superimposed on entropy contours. The right side shows Mach contours. The supersonic zone surrounding the vehicle is much better resolved with the adapted grid. The size of the vortex structures on the top of the vehicle is improved due to adaptation. However, the complexity of the near surface flow is still not well captured with the adapted coarse grid. 


\section{Conclusions}

Four related grids were generated for Navier-Stokes calculations on the Ares I a105 OML configuration. In general, changes in $C_{p}$ become smaller with grid refinement but convergence in pressure coefficient has not been demonstrated even in the fine grid. The changes suggested that the baseline node spacing is probably adequate on the clean areas of the first and upper stages. Increments in $C_{p}$ indicated that the causes of the lack of convergence are located in the flow field near the frustum region and near the aft skirt on the first stage at maximum dynamic pressure and also in the crew module area at near sonic speed. These areas are where the shock is located as the Mach number progresses through the transonic regime. Separated flow regions change character, often between the coarse and baseline or baseline and fine grids. Feature-based, adapted coarse grid results recovered many flow field features found on the fine grid which were not captured by the original coarse grid.

The present analysis clearly indicates that strong velocity and Mach number gradients can occur on the vehicle surface at any location, irrespective of the proximity of protuberances or geometry slope discontinuities. Successively refined solutions show that additional grid refinement between the interstage/frustum junction and the first stage tumble motors changes the location of the shock separation relative to the stagnation ahead of the protuberance. A trade-off between resolving protuberances sufficiently and capturing flow features not related to protuberances complicates the effort of grid resolving a complex geometry through the transonic flow regime. But it does justify a strategy of adding generally more grid refinement at any location on the body at which strong gradients are expected. This study has clearly shown that grid adaptation is needed to fully resolve flow features about the complex geometry of the Ares I vehicle.

The strategy of starting from the coarse grid and limiting the number of nodes added by the grid adaptation steps, and using mainly moving of nodes and realignment, has yielded some improvement in solutions. However, the approach taken here of primarily realigning element edges/faces with off body flow features with minimal node addition alone does not sufficiently improve the flow modeling near surfaces. It is possible that adapting the fine grid would produce a significantly better solution. If the approach is taken, as was done here, to start with a coarse grid, it is clear that an additional capability to refine the boundary layer and surface grid is needed.

\section{Acknowledgments}

The authors would like to acknowledge Pat Cosgrove and Paresh Parikh of the Ares Project Office for their support of this work. The authors wish to thank Steve Massey of the Aeroelasticity Branch, NASA Langley Research Center for providing the interpolation software used in this study.

\section{References}

\footnotetext{
${ }^{1}$ Dotson, K. and Engblom, W. A., "Vortex-Induced Vibration of a Heavy-lift Launch Vehicle During Transonic Flight," Journal of Fluids and Structures, Vol. 19, 2004, pp. 669-680.

${ }^{2}$ Engblom, W. A., "Numerical Simulation of Titan IVB Transonic Buffet Environment," Journal of Spacecraft and Rockets, Vol. 40, 2003, pp. 648-656.

${ }^{3}$ Venditti, D. A. and Darmofal, D. L., "Grid Adaptation for Functional Outputs: Application to Two-Dimensional Inviscid Flows," Journal of Computational Physics, Vol. 176, 2002, pp. 40-69.

${ }^{4}$ Venditti, D. A. and Darmofal, D. L., "Anisotropic Grid Adaptation for Functional Outputs: Application to Two-Dimensional Viscous Flows," Journal of Computational Physics, Vol. 187, 2003, pp. 22-46.

${ }^{5}$ Lee-Rausch, E. M., Park, M. A., Jones, W. T., Hammond, D. P., and Nielsen, E. J., "Application of a Parallel Adjoint Based Error Estimation and Anisotropic Grid Adaptation for Three-Dimensional Aerospace Configurations," 23rd AIAA Applied Aerodynamics Conference, No. 4842, 2005.

${ }^{6}$ Park, M. A., “Adjoint-Based Three-Dimensional Error Prediction and Grid Adaptation,” AIAA Journal, Vol. 42, 2004, pp. $1854-1862$.

${ }^{7}$ Casper, J. H., Cliff, S. E., Thomas, S. D., Park, M. A., McMullen, M. S., Melton, J. E., and Durston, D. A., "Assessment of Near-Field Sonic Boom Simulation Tools," 26th AIAA Applied Aerodynamics Conference, No. 6592, 2008.

${ }^{8}$ Park, M. A., "Three-Dimensional Turbulent RANS Adjoint-Based Error Correction," 16th AIAA Computational Fluid Dynamics Conference, No. 3849, 2003.

${ }^{9}$ Bibb, K. L., Gnoffo, P. A., Park, M. A., and Jones, W. T., "Parallel, Gradient-based, Anisotropic Mesh Adaptation for Re-entry Vehicle Configurations," 9th AIAA/ASME Joint Thermophysics and Heat Transfer Conference, No. 3579, 2006.

${ }^{10}$ Bigarella, E. D. V., Basso, E., and Azevedo, J. L. F., "Multigrid Adaptive-Mesh Turbulent Simulations of Launch Vehicle Flows," 21 st AIAA Applied Aerodynamics Conference, No. 4076, 2003.

${ }^{11}$ Scalabrin, L. C. and Azevedo, J. L. F., "Three Dimensional Flow Simulations with the Finite Element Technique over a Multi-Stage Rocket," 40th AIAA Aerospace Sciences Meeting and Exhibit, No. 0408, 2002.

${ }^{12}$ Scalabrin, L. C. and Azevedo, J. L. F., "Hierarchical Mesh Refinement and Coarsening for Unstructured Finite Volume Flow Simulations," Proceedings of the 5th World Congress on Computational Mechanics, Vienna, Austria, 2002.

${ }^{13}$ Scalabrin, L. C. and Azevedo, J. L. F., "Finite Volume Launch Vehicle Flow Simulations on Unstructured Adaptive Meshes," 41 st AIAA Aerospace Sciences Meeting and Exhibit, No. 601, 2003.
} 
${ }^{14}$ Strauss, D. and Azevedo, J. L. F., "Unstructured Multigrid Simulations of Turbulent Launch Vehicle Flows Including a Propulsive Jet," Journal of Spacecraft and Rockets, Vol. 41, No. 5, 2004, pp. 745-753.

${ }^{15}$ Park, M. A. and Carlson, J.-R., "Turbulent Output-Based Anisotropic Adaptation," 48th AIAA Aerospace Sciences Meeting, No. $168,2010$.

${ }^{16}$ Bartels, R. E., Chwalowski, P., Massey, S., Heeg, J., Wieseman, C., and Mineck, R., "Computational aeroelastic analysis of the Ares launch vehicle during ascent," 28th AIAA Applied Aerodynamics Conference, No. 4374, 2010.

${ }^{17}$ Anderson, W. K., Rausch, R. D., and Bonhaus, D. L., "An Implicit Upwind Algorithm for Computing Turbulent Flows on Unstructured Grids," Computers and Fluids, Vol. 23, No. 1, 1994, pp. 1-22.

${ }^{18}$ NASA LaRC, Hampton, VA, FUN3D Manual, November 2008, http: / / fun3d. larc. nasa.gov.

${ }^{19}$ Spalart, P. R. and Allmaras, S. R., "One-Equation Turbulence Model for Aerodynamic Flows," 30th AIAA Aerospace Sciences Meeting and Exhibit, No. 439, 1992.

${ }^{20}$ Vatsa, V. N. and White, J. A., "Calibration of a Unified Flux Limiter for Ares-Class Launch Vehicles from Subsonic to Supersonic Speeds," 56th Propulsion Meeting 5, JANNAF, III, April 2009.

${ }^{21}$ Nielsen, E. J., Lu, J., Park, M. A., and Darmofal, D. L., "An Exact Dual Adjoint Solution Method for Turbulent Flows on Unstructured Grids," Computers and Fluids, Vol. 33, No. 9, 2004, pp. 1131-1155.

${ }^{22}$ Park, M. A., Anisotropic Output-Based Adaptation with Tetrahedral Cut Cells for Compressible Flows, Ph. D. thesis, Massechusetts Institute of Technology, 2008.

${ }^{23}$ Tecplot, Inc., Tecplot, 2010, http: //www.tecplot.com.

${ }^{24}$ Kennel, M. B., "KDTREE 2: Fortran 95 anc C++ Software to Efficiently Search for Near Neighbors in a Multi-Dimensional Euclidean Space," e-prints:arXiv.org, Cornell University Library, August 2004. 
Table 1. CFD test conditions.

\begin{tabular}{ccccc}
\hline \hline time, sec. & altitude, ft. & Mach No. & $\alpha$, deg. & Rey. No., per inch \\
\hline 22.6 & 5796 & 0.50 & 5.0 & 253233 \\
37.7 & 16592 & 0.90 & 2.5 & 336057 \\
56.6 & 36433 & 1.55 & 2.5 & 305090 \\
\hline \hline
\end{tabular}

Table 2. Grids for Grid Refinement Studies

\begin{tabular}{cccc}
\hline \hline Grid & Surface Nodes (Millions) & Viscous Nodes (Millions) & Total Nodes (Millions) \\
\hline Extra Coarse & 0.299 & 8.55 & 9.75 \\
Coarse & 0.556 & 15.58 & 19.00 \\
Baseline & 1.114 & 30.54 & 40.84 \\
Fine & 2.075 & 55.62 & 83.37 \\
\hline \hline
\end{tabular}

Table 3. Adapted Coarse Grid Size.

\begin{tabular}{cc}
\hline \hline Adaptation & Total Nodes (Millions) \\
\hline original & 19.00 \\
1 & 18.79 \\
2 & 18.86 \\
3 & 18.96 \\
4 & 19.17 \\
5 & 19.52 \\
6 & 20.08 \\
7 & 20.89 \\
\hline \hline
\end{tabular}

9 of 16 


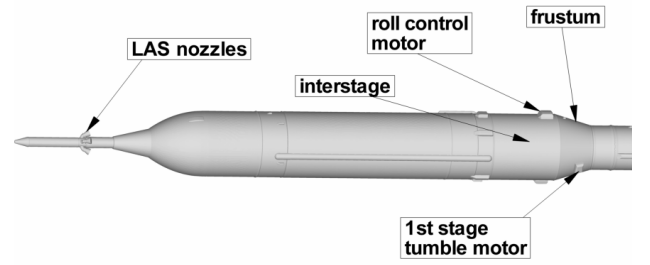

(a) Upper stage

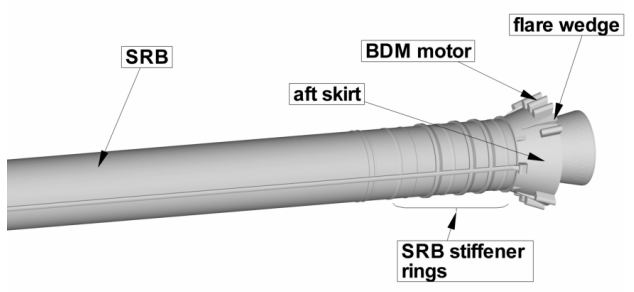

(b) First stage

Figure 1. Drawing of the Ares I a105 OML.

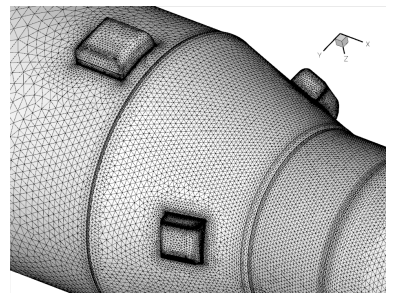

(a) Extra coarse grid.

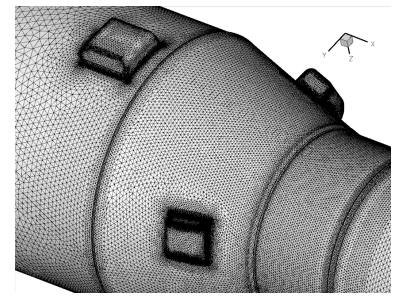

(b) Coarse grid.

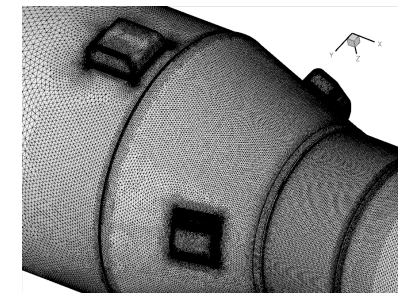

(c) Baseline grid.



(d) Fine grid.

Figure 2. Examples of the surface grid near the frustum.

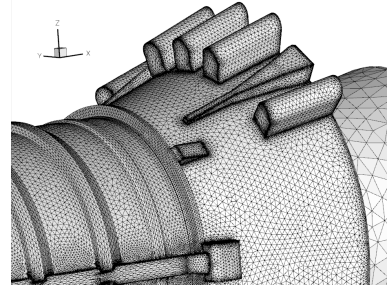

(a) Extra coarse grid.

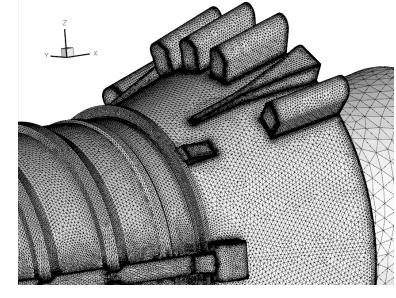

(b) Coarse grid.

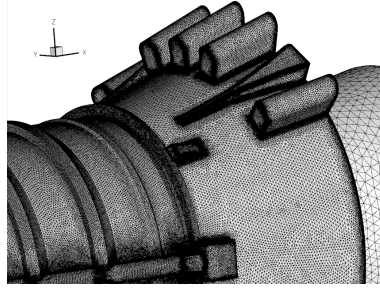

(c) Baseline grid.

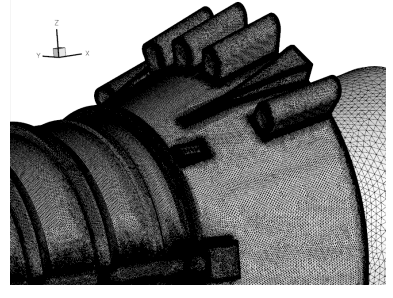

(d) Fine grid.

Figure 3. Examples of the surface grid near the skirt at the base of the first stage.

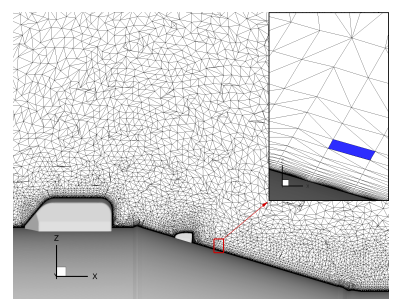

(a) Extra coarse grid.

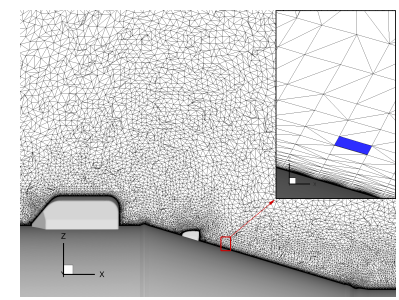

(b) Coarse grid.

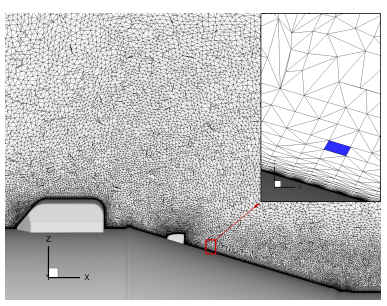

(c) Baseline grid.

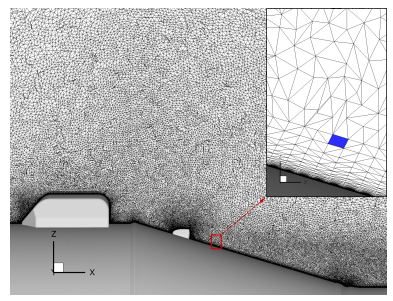

(d) Fine grid.

Figure 4. Examples of the off-body grid near the frustum.



(a) Extra coarse grid.

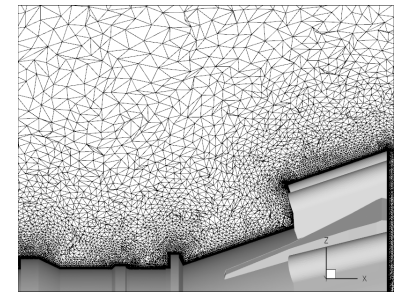

(b) Coarse grid.

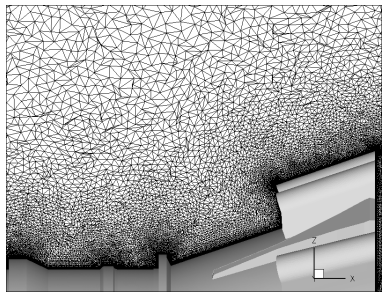

(c) Baseline grid.

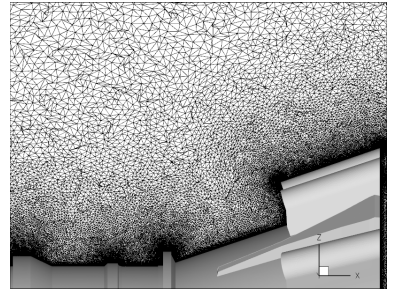

(d) Fine grid.

Figure 5. Examples of the off-body grid near the base of the first stage. 


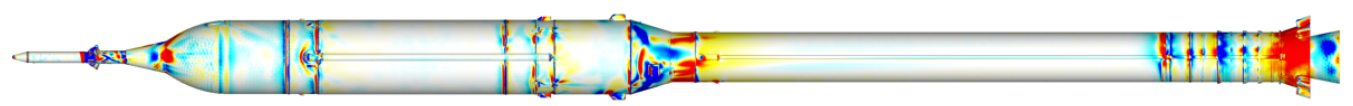

(a) Extra coarse to fine grid.

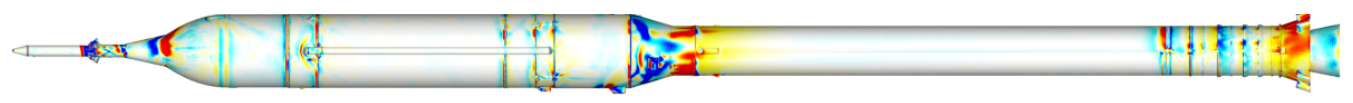

(b) Coarse to fine grid.

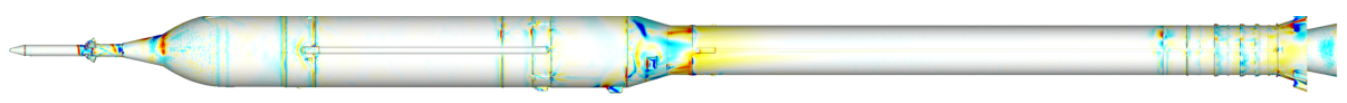

(c) Baseline to fine grid.

Figure 6. Difference in surface $C_{p}$ contours relative to the fine grid. $M=1.55$

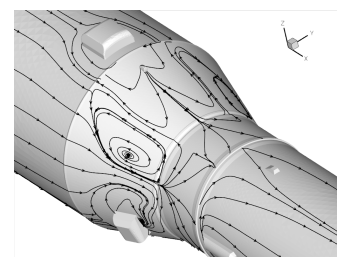

(a) Extra coarse grid.



(b) Coarse grid.

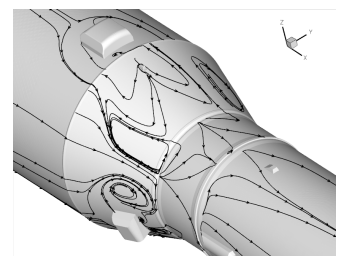

(c) Baseline grid.

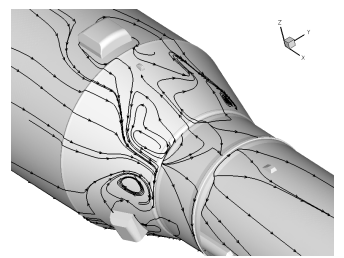

(d) Fine grid.

Figure 7. Surface streamlines over frustum at $M=1.55$.

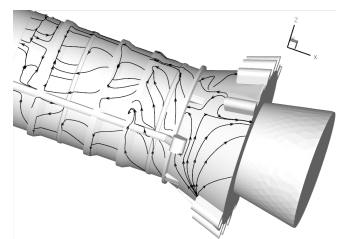

(a) Extra coarse grid.

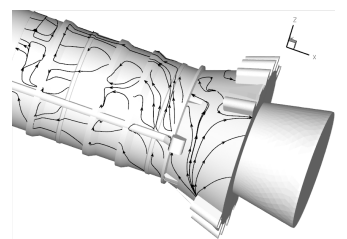

(b) Coarse grid.

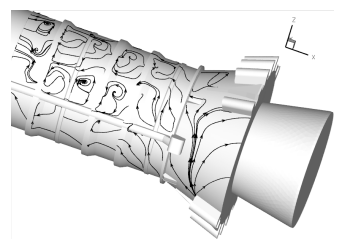

(c) Baseline grid.

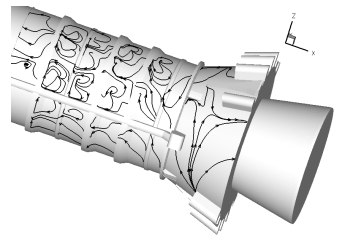

(d) Fine grid.

Figure 8. Surface streamlines on the lower portion of the first stage at $M=1.55$. 


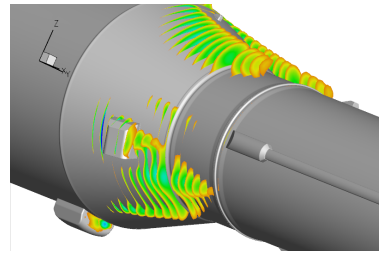

(a) Extra coarse grid.

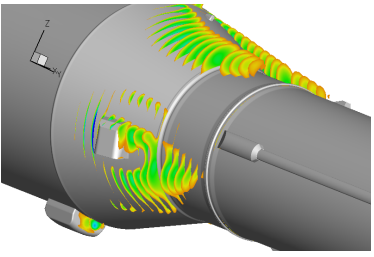

(b) Coarse grid.



(c) Baseline grid.

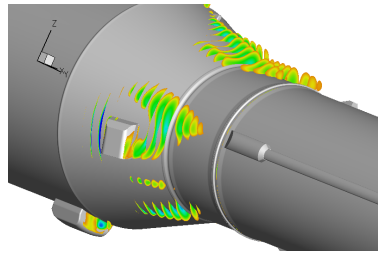

(d) Fine grid.

Figure 9. Negative streamwise velocity contours over the frustum at $M=1.55$.

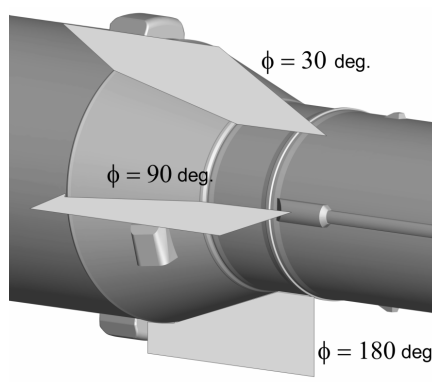

Figure 10. Clocking angles of frustum contour cuts

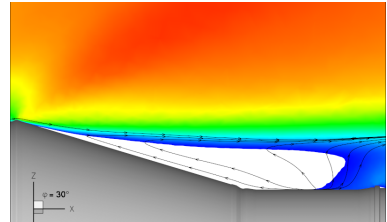

(a) Extra coarse grid.

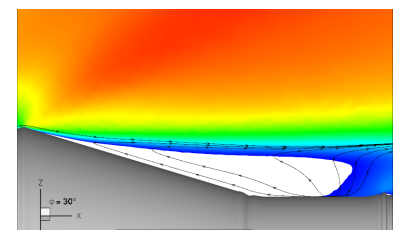

(b) Coarse grid.

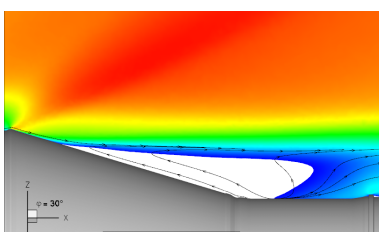

(c) Baseline grid.

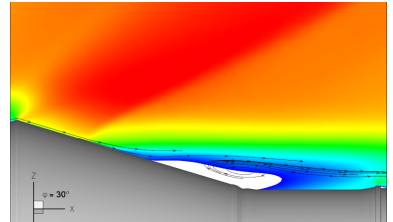

(d) Fine grid.

Figure 11. Streamwise velocity contours over the frustum. $\phi=30^{\circ}$ at $M=1.55$.(red - max, blue - min, plus only)

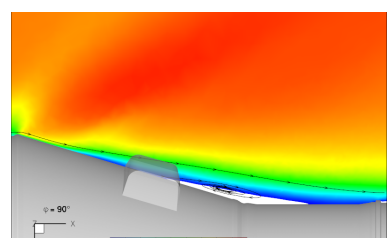

(a) Extra coarse grid.

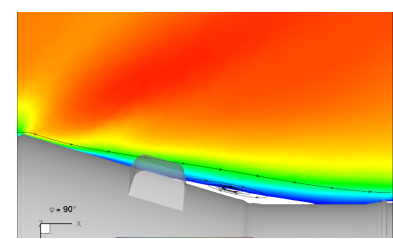

(b) Coarse grid.

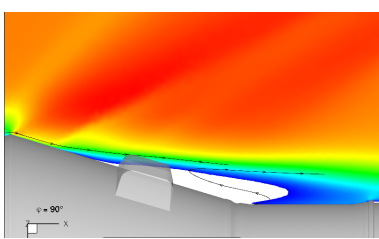

(c) Baseline grid.

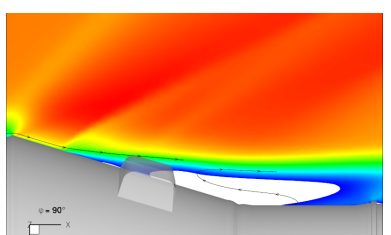

(d) Fine grid.

Figure 12. Streamwise velocity contours over the frustum. $\phi=90^{\circ}$ at $M=1.55$.(red - max, blue - min, plus only)

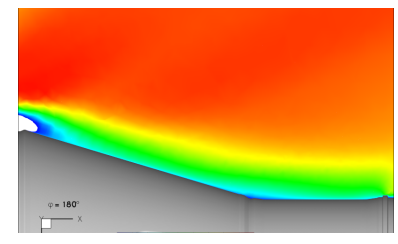

(a) Extra coarse grid.

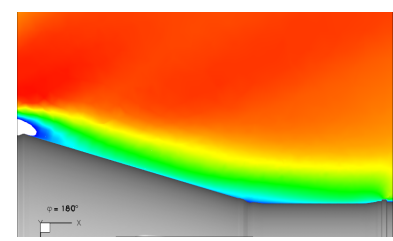

(b) Coarse grid.

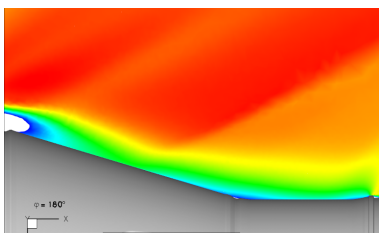

(c) Baseline grid.



(d) Fine grid.

Figure 13. Streamwise velocity contours over the frustum. $\phi=180^{\circ}$ at $M=1.55$.(red - max, blue - min, plus only) 


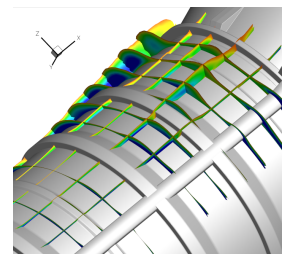

(a) Extra coarse grid.

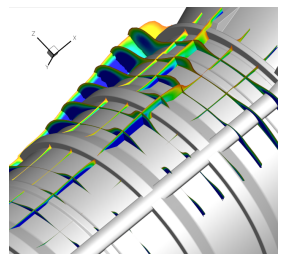

(b) Coarse grid.

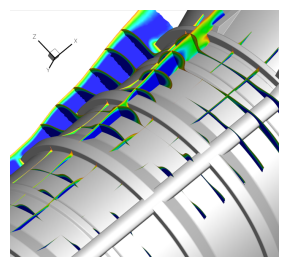

(c) Baseline grid.



(d) Fine grid.

Figure 14. Contours of negative streamwise velocity over the wide rings near the first stage aft skirt at $M=1.55$.

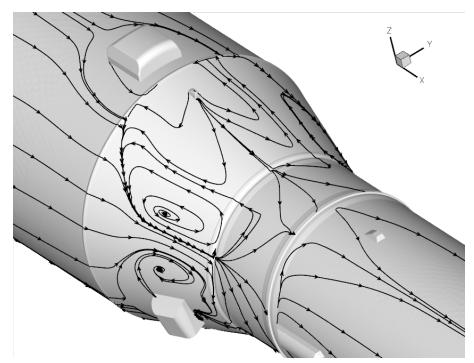

(a) Original coarse grid.



(b) Adapted grid.

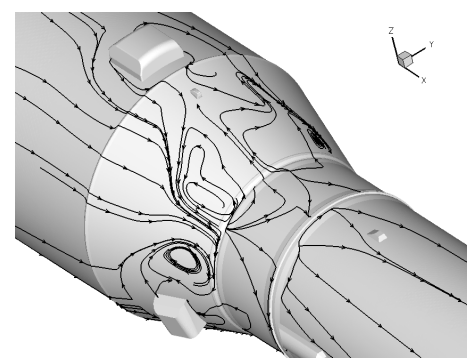

(c) Fine grid.

Figure 15. Surface streamlines over the frustum on the original coarse, the adapted and the fine grids at $M=1.55$.

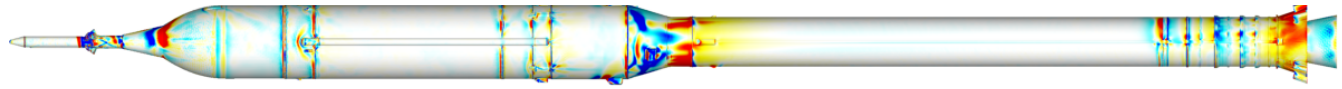

(a) Original Coarse to fine grid.

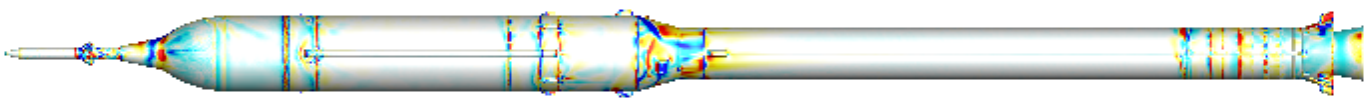

(b) Adapted coarse to fine grid.

Figure 16. Difference in surface $C_{p}$ contours between original coarse, the adapted and fine grids at $M=1.55$. 


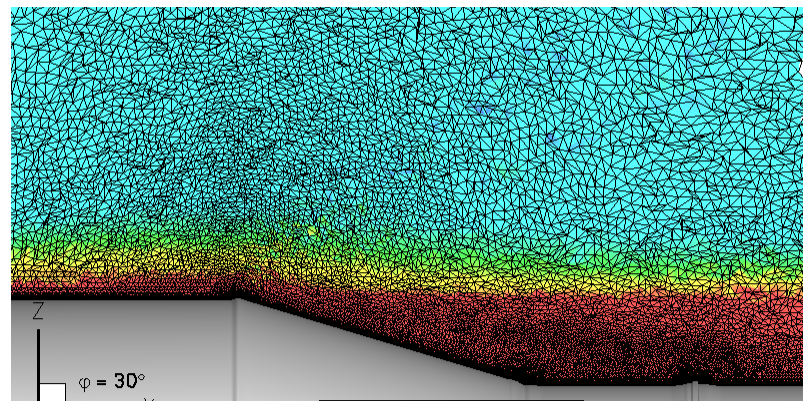

(a) Original coarse (entropy)

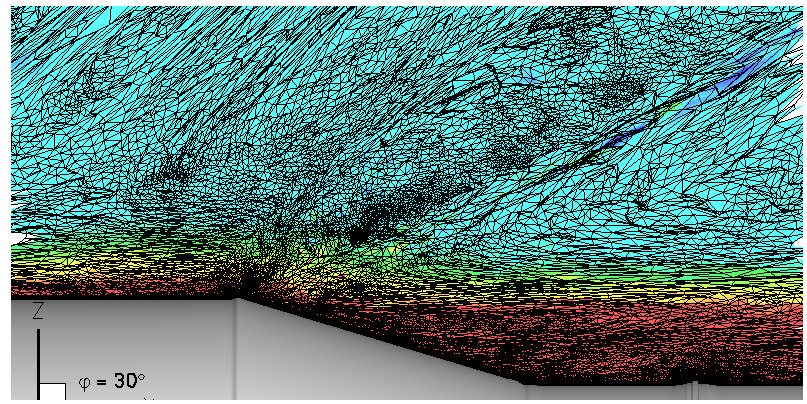

(c) Adapted coarse (entropy).

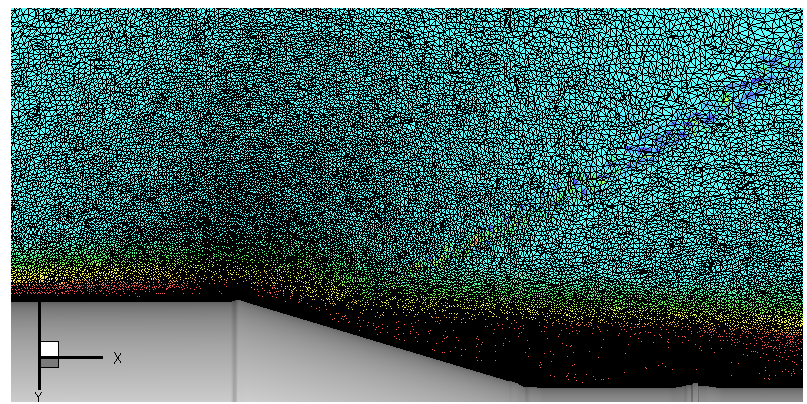

(e) Original fine (entropy).

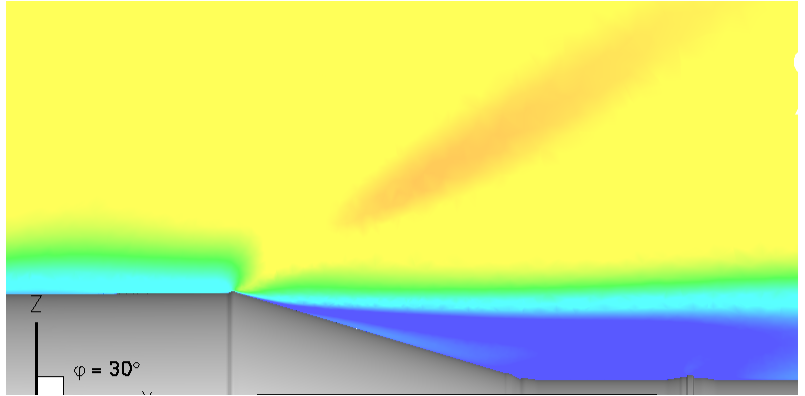

(b) Original coarse (Mach).

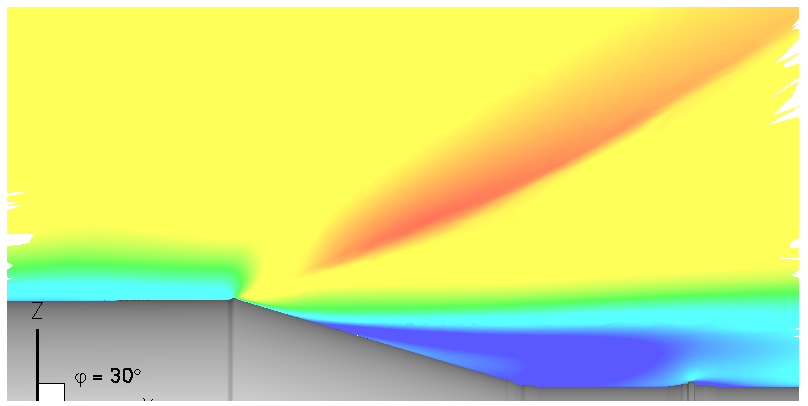

(d) Adapted coarse (Mach).

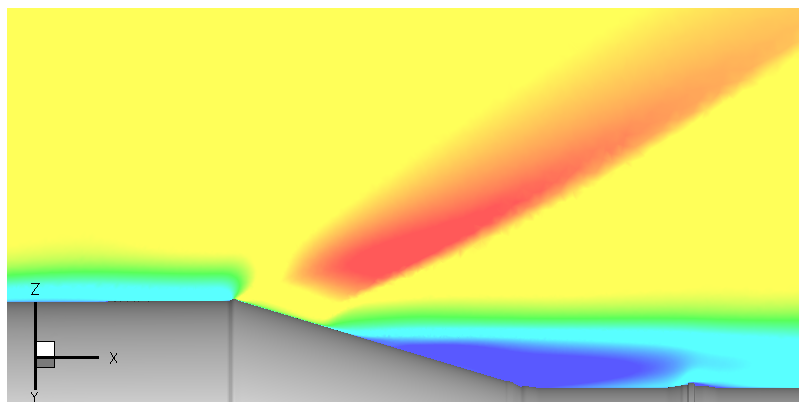

(f) Original fine (Mach).

Figure 17. Contours over frustum, $\phi=30^{\circ}$ for the original coarse, the adapted, and the fine grids at $M=1.55$.(red - max, blue - min) 


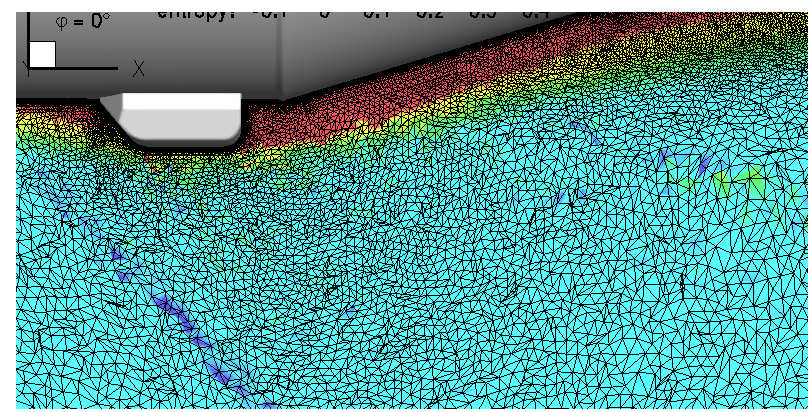

(a) Original coarse (entropy).

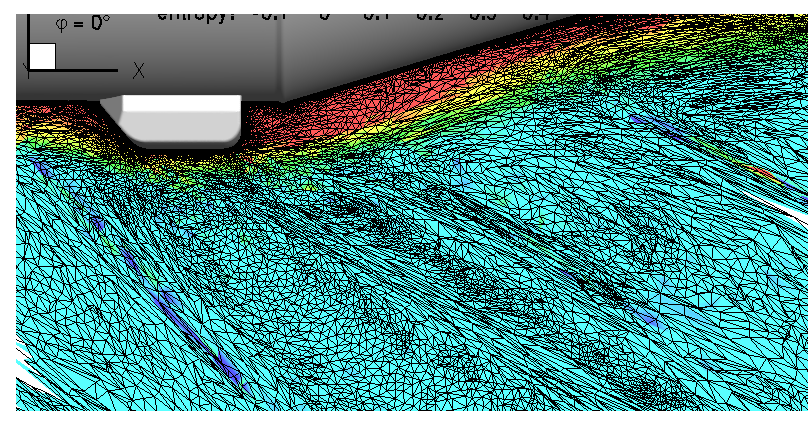

(c) Adapted coarse (entropy).

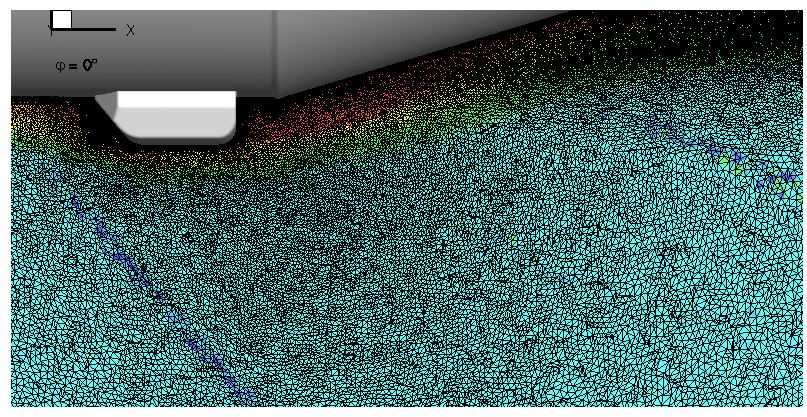

(e) Original fine (entropy).

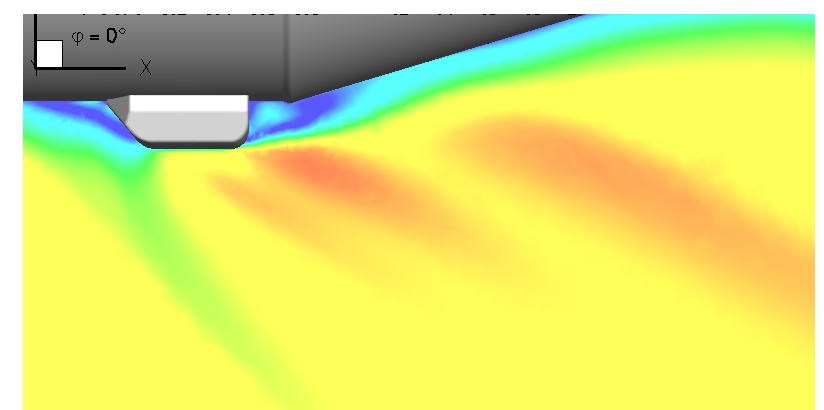

(b) Original coarse (Mach).



(d) Adapted coarse (Mach).

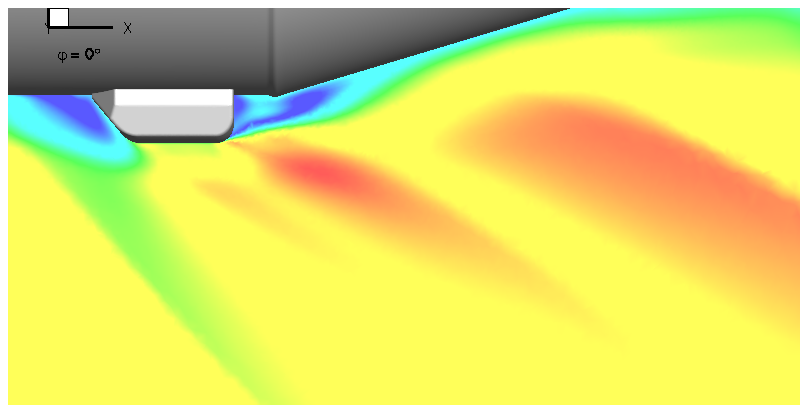

(f) Original fine (Mach).

Figure 18. Contours over frustum, $\phi=180^{\circ}$ for the original coarse, the adapted, and the fine grids at $M=1.55 .($ red - max, blue - min) 


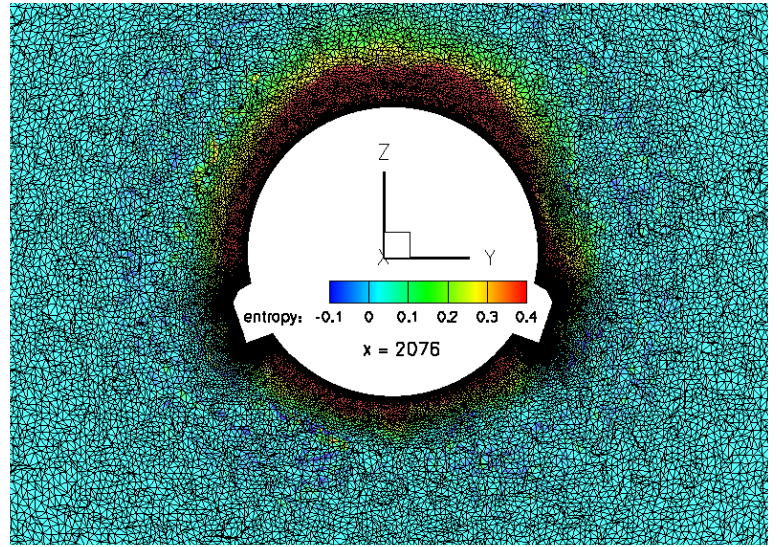

(a) Original coarse (entropy).

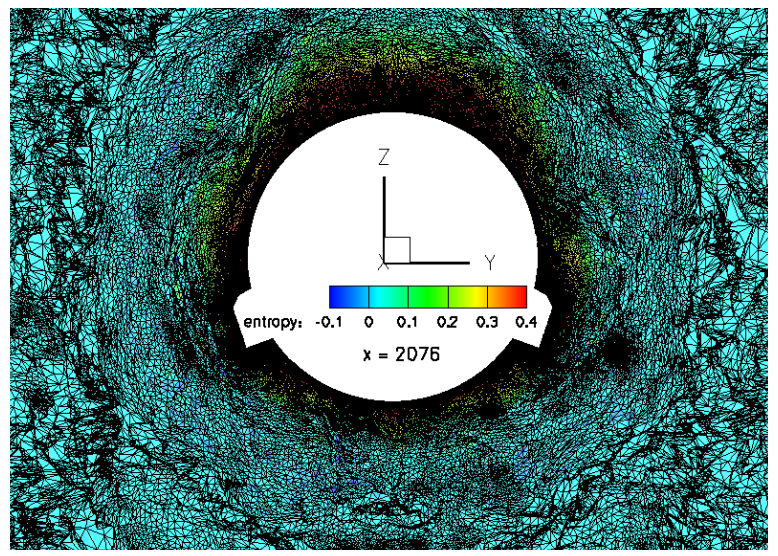

(c) Final adapted coarse (entropy).

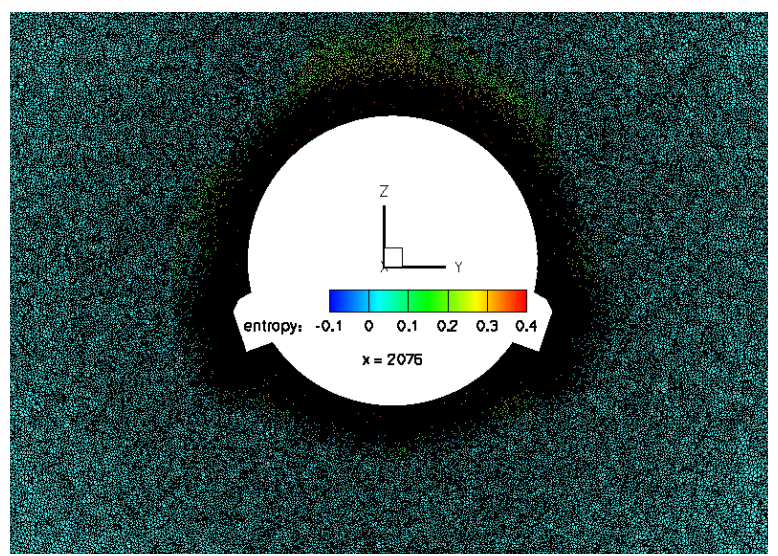

(e) Original fine (entropy).

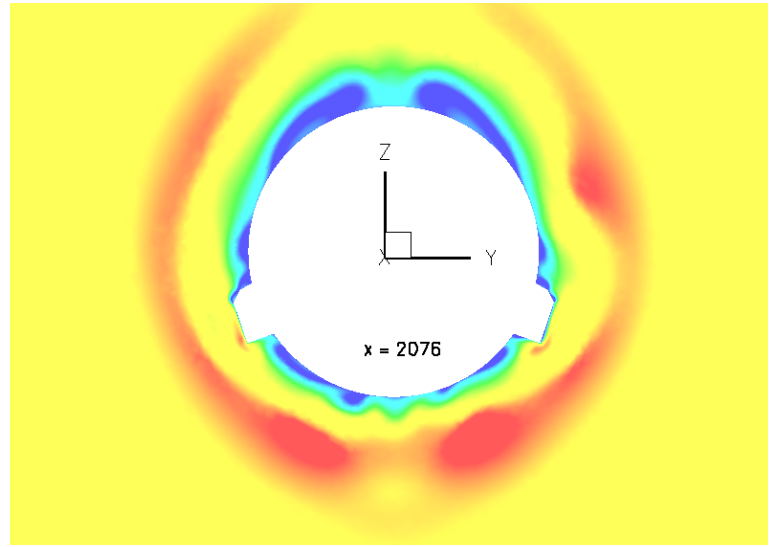

(b) Original coarse (Mach)

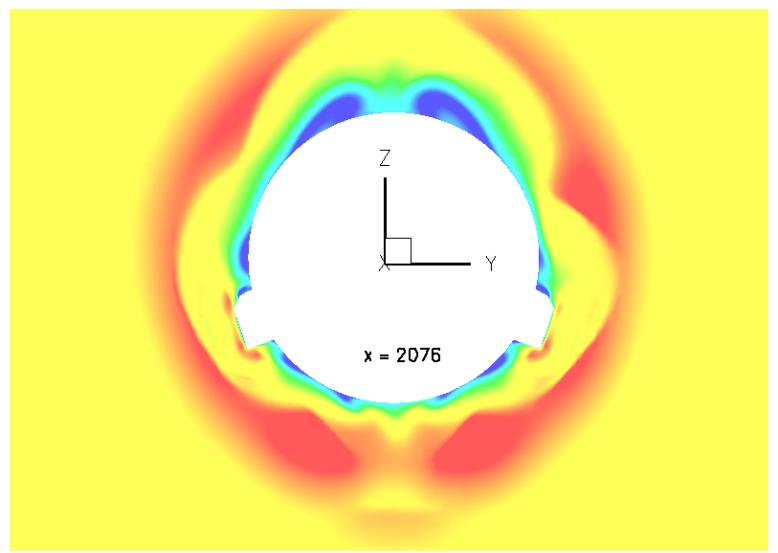

(d) Final adapted coarse (Mach).

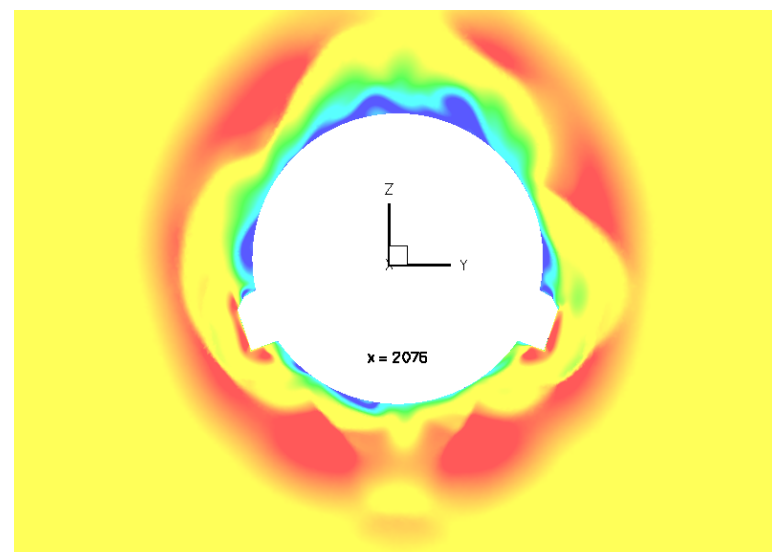

(f) Original fine (Mach).

Figure 19. Off-body grid and flow field data on a slice perpendicular to the vehicle at $\mathbf{x}=\mathbf{2 0 7 6}$ for the original coarse, the adapted, and the fine grids at $M=1.55$.(red - max, blue - min) 